\title{
Natural antidote to global warming?
}

The condition of the Sun in the seventeenth century suggests that reduced radiation could have accounted for the Little Ice Age, but there is only a small chance that a recurrence will head off global warming.

Could an engineered return of the Maunder minimum be a credible antidote to the threat of global warming in coming decades? Speculations of that kind naturally have no place in the real world of practical politics, with its carbon taxes and so on, but they are more substantial than mere nostalgia. What is the chance that the Sun will save our bacon by lapsing into a condition free from sunspots? And would that be enough to offset the effects of greenhouse gases in the atmosphere?

The Maunder minimum is the period from 1645 to 1715 , close on the heels of Galileo's first observation of apparently black spots on the surface of the Sun. Throughout the Maunder minimum, there were no spots or other signs of surface activity on the Sun. Famously, the Maunder minimum came towards the end of what was known in the Northern Hemisphere as the Little Ice Age (spanning four centuries from 1450). In London, the surface of the Thames used regularly to freeze in the winter, encouraging novel forms of party-going. European literature of the period is replete with images of intense winter cold.

How would the lack of sunspots (and of other forms of activity) have affected the climate on the surface of the Earth? If there were a better understanding of what happens in the outer layers of the Sun, the answer might tumble out from a calculation of some kind, but that is not possible. But old-fashioned inference can accomplish a great deal. Ingeniously, J. L. Lean from the US Naval Research Laboratory and $O$. R. White and A. Skumanich from the National Center for Atmospheric Research at Boulder, Colorado, have now used a variety of proxies for solar activity to estimate both the output of energy from the Sun and its spectral distribution during the Maunder minimum (Glob. biogeochem. Cycles 9, 171-182; 1995).

The chain of inference is elaborate, but all the more interesting on that account. How can one estimate sunspot numbers at times before people knew there were such things? The rate of production of cosmogenic radioactive isotopes, such as ${ }^{14} \mathrm{C}$ and ${ }^{10} \mathrm{Be}$, by the interaction of cosmic rays with atomic nuclei in the upper atmosphere is one good proxy, because the intensity of cosmic rays reaching the Earth is modulated by the activity of the Sun. And how is it possible to infer the output of ultraviolet radiation from the Sun during the Maunder minimum when accurate records of ultraviolet radiation (based on satellite measurements) are available only since about 1980? Use observations of other stars like the Sun as a guide, of course.

The isotope data were first decisively used by J. A. Eddy (Science 192, 1189-1202; 1976). The essence of the technique is that the Earth is partially shielded from (comparatively) low-energy cosmic rays by an active Sun, with the result that cosmogenic isotope production is then decreased. The result is that the Maunder minimum is recognizable by extra ${ }^{12} \mathrm{C}$ in tree-rings and extra ${ }^{10} \mathrm{Be}$ in ice-cores from the period. Lean and his colleagues echo Eddy's original ambition that a careful comparison of past isotope production would allow a detailed reconstruction of the Sun's activity in the past, but evidently the data are not yet good enough to do more than identify a rather longer minimum beginning in the fourteenth century.

The other leg of the chain of inference is the use of contemporary records of ultraviolet emission from the Sun and observations of stars similar in mass and age to the Sun to reconstruct the ultraviolet spectrum of the Sun during the Maunder minimum. The crucial data are those due to Salie Baliunas and Robert Jastrow (Nature 348, 520-523; 1990), who hunted through Palomar and Mount Wilson plates to find a sample of stars like the Sun and for evidence of their ultraviolet emission in bands due to hydrogen and potassium, taken as proxies for magnetic activity. Their conclusion was that some stars have magnetic cycles like the Sun, and that others do not.

The objective, as stated, is to estimate the ultraviolet output of the Sun during the Maunder minimum. Lean et al. first show that there is a tight correlation between the Lyman- $\alpha$ emission line, the He 1083-nm emission lines and the $\mathrm{Mg}^{+}$ absorption line with the $\mathrm{Ca}^{+}$absorption or Fraunhofer features of the solar spectrum. That allows them to take recent measurements of the $\mathrm{Ca}^{+}$index as a measure of total ultraviolet irradiance. To estimate ultraviolet emission during the Maunder minimum, it is then simply necessary to use the measured $\mathrm{Ca}^{+}$emission from stars like the Sun, but showing no magnetic activity, to represent what the real Sun must have been doing in the seventeenth century.
The outcome is not on the face of things sensational. The total irradiance of the Sun during the Maunder minimum would have been 0.2 per cent less than that of the present quiet Sun, but the decrease of ultraviolet flux would have been much greater. Thus Lyman- $\alpha$ radiation would have been reduced by no less than 64 per cent (compared with the quiet Sun) during the Maunder minimum. Total ultraviolet output would have been more than 1 per cent less.

So does the altered output of energy from the Sun account for the severe winters of the Maunder minimum? Lean et al. remark that a global model of the climate predicts a temperature decline of $0.46{ }^{\circ} \mathrm{C}$ for a decrease of solar irradiance by 0.25 per cent, which is in itself a large fraction of the estimated average cooling (between 0.5 and $1.0^{\circ} \mathrm{C}$ ) during the Little Ice Age. They would have been even nearer the mark if they had followed the notion that the diameter of the Sun decreases during quiet spells (which should be measurable at the epoch free of sunspots).

The prolonged absence of sunspots would nevertheless have affected the ozone column in the atmosphere. On the basis of what they have learned about the reduction of ultraviolet flux, Lean et al. say that the total ozone column may have been reduced by between 4 and 8 per cent. With luck, that may account for the largest estimates of mean temperature reduction during the Little Ice Age. But nothing is as yet certain.

There remains the question of whether the next Maunder minimum will arrive in time to avoid a global carbon tax. Lean $e t$ $a l$. have nothing to say on the subject, but there is a clue in the article by Baliunas and Jastrow, who say that roughly a third of a sub-sample of their Sun-like stars appeared to be magnetically quiet at any time. Allowing for the Maunder minimum and earlier and longer events centred on the fifteenth century, it seems that our own Sun has been spot-free for about a third of this millennium.

The statistics are not yet good enough to suggest how long climatic minima last, or how long are the intervals between them, but if the crunch-time for global warming comes in around 2050, the five solar cycles between now and then hardly seem enough to ensure safety. The moral: a better understanding of the Sun might now have practical value.

John Maddox 